WS12-B02

\title{
Tomographic Velocities - Challenges and Applications
}

\author{
L. Vigée* (CGG), M. Bader (CGG), A. Bénédini (SCDM-ENERGIE), C. Brillatz \\ (CGG), D. Carotti (CGG), A. Cavalié (CGG), T. Coléou (CGG), P. Guillaume \\ (CGG), G. Hénin (CGG), G. Lambaré (CGG), T. Le Ruyet (CGG), L. Lopes \\ (CGG), A. Vasseur (CGG) \& N. Vidal (CGG)
}

\section{SUMMARY}

The resolution of seismic imaging has long been characterized by a mid-frequency gap between the long vertical wavelength components that can be inferred from travel-time tomography and the short vertical wavelength components that can be inferred from seismic migration. The progress in tomography and in broadband acquisition now allows this mid-wavelength gap to be filled and even results in the overlapping between the resolution obtained from velocity model building and from seismic migration. Recent progress in tomographic approaches now provides vertical resolution up to $6 \mathrm{~Hz}$, with in addition, a precise localization of the velocity contrasts. With a case study we discuss their benefits for reservoir characterization, where the missing frequencies are traditionally coming from a model interpolated/ extrapolated from well log information. If the seismic inversion for reservoir characterization is not sensitive to the low-to-mid frequencies, this low-to-mid frequency modelling is critical for the estimation of absolute rock properties, and replacing a model extrapolated from sparse well information by actual measurements is of paramount importance. 


\section{Amsterdam ' 14}

\section{Introduction}

Claerbout (1985) highlighted a mid-frequency gap (2 to $10 \mathrm{~Hz}$ ) in the reliability of information obtained from surface seismic measurement, and corresponding to the gap between the long vertical wavelength components that can be inferred from travel-time tomography and the short wavelength components that can be inferred from the traces through the depth migration process.

With progress in broadband acquisition and processing we are now at a point where the midfrequency gap can be eliminated. We emphasize here filling the gap using the results of modern raybased tomography methods and the resulting benefits for reservoir characterization. For initial model building prior to seismic inversion, the missing frequencies are traditionally filled using a model interpolated/extrapolated from low-pass filtered well log information. This low-to-mid frequency information is critical for quantitative estimation of the rock properties, and velocity models obtained by modern ray-based tomography methods are providing some of this information.

\section{Filling the mid-frequency gap with ray-based tomography}

Two types of tomographic approaches have recently established the high-resolution seismic interval velocities as a more valid attribute for reservoir characaterization. The first one allows sharp, geologically-consistent, velocity contrasts in the velocity model (multi-layer non-linear slope tomography proposed by Guillaume et al. (2013)), the second one uses dense volumetric picking (high definition tomography proposed by Guillaume et al. (2012)).

The introduction of sharp contrasts in the velocity model as well as the conversion of dense residual moveout information into a high-resolution structurally conformant velocity model greatly enhance the vertical resolution and the relevance of velocity for reservoir characterization. Thus, tomography seriously complements full waveform inversion especially considering that it can interpret the kinematics of reflected waves and that it can resolve velocities with a vertical resolution up to $6 \mathrm{~Hz}$.

\section{Application for reservoir characterization}

As seismic is band-limited, seismic inversion results are often considered to be insensitive to the initial model and to the modelling choices that were made, i.e. they are unable to update any low-tomid frequency structure present in the initial model. This is no longer true with the increased resolution of the velocity information that includes frequencies that are within the reflectivity band and with the presence of steps in the velocity field corresponding to accurate positioning of the major velocity discontinuities. These velocity contrasts provide a first-order description of the velocity changes across existing lithological boundaries with their corresponding reflectivity that is absent from low frequency trends obtained by filtering well $\operatorname{logs}$ and spatial extrapolation. Figure 1 shows sections from different velocity models obtained by multi-layer non-linear slope tomography.

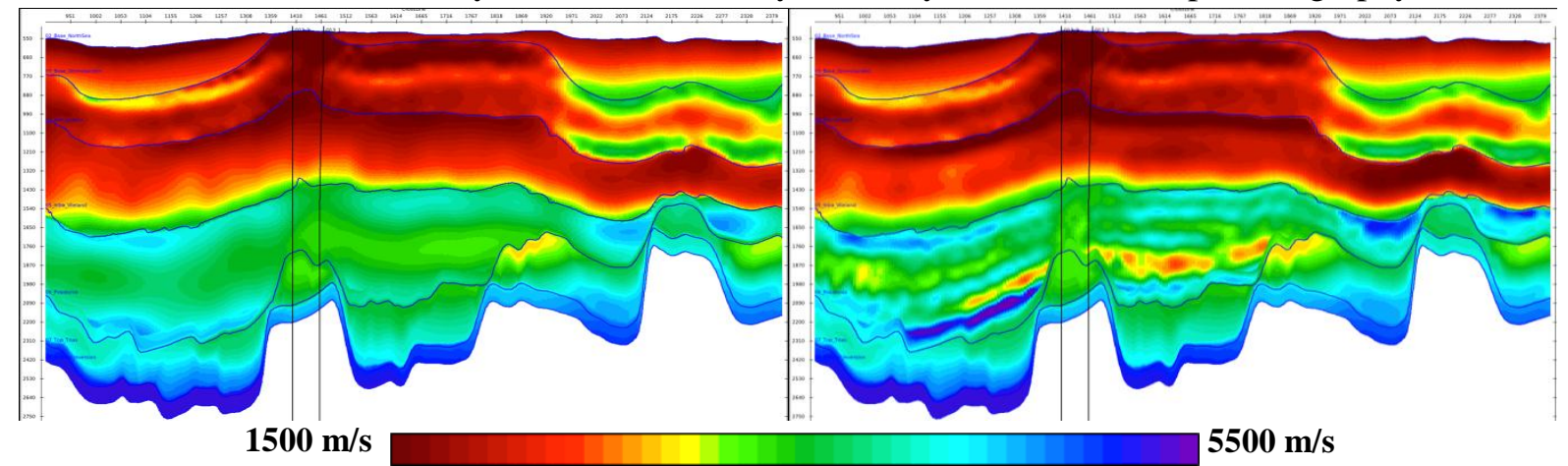

Figure 1: Velocity models with sharp contrasts obtained by multi-layer non-linear slope tomography: (multi-layer tomography left) and with the addition of high definition tomography (right).

Such velocity models are more suitable initial models for seismic reservoir characterization. To validate this, the inversions are performed with tight constraints on possible changes from the initial model, allowing us to measure how good a match can be obtained with minimal changes and to compare how close each model is to explaining the seismic reflectivity. In Figure 2, the same velocity sections after constrained seismic inversion are shown.

The Quality Indicator (QI) (Coléou al., 2013) is a repeatability attribute derived from Correlation and NRMS. It is used to quantify the match between seismic and synthetic traces after seismic inversion. 


\section{Amsterdam ' 14}

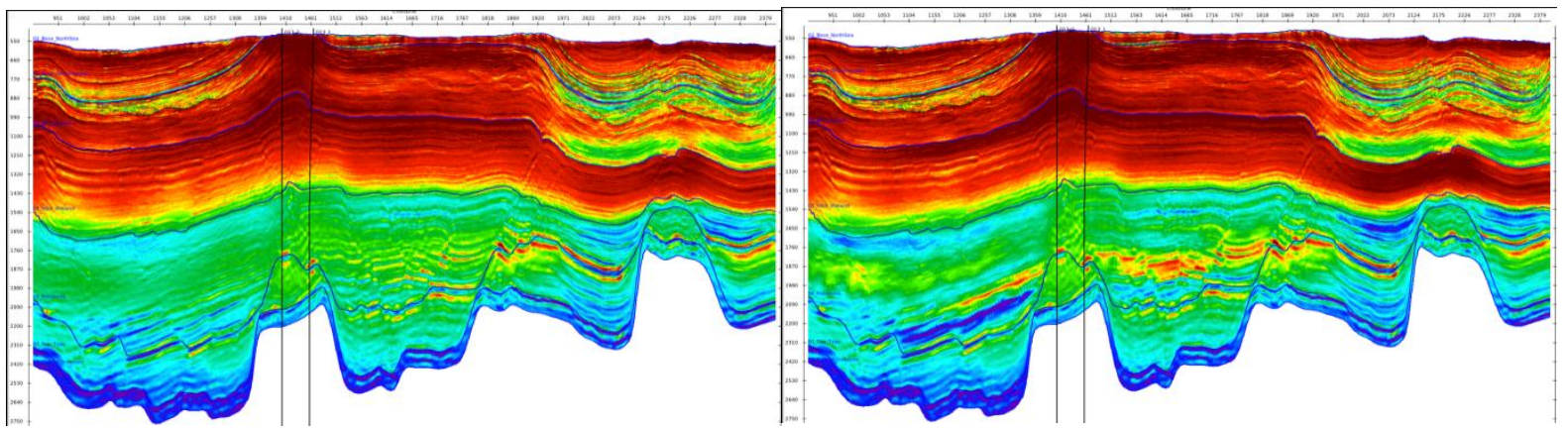

Figure 2: Velocity models after constrained seismic inversion derived from multi-layer tomography (left) and multi-layer tomography + high definition tomography (right)

In Figure 3, we see results for the PSTM low-frequency model (in pink) overlaying the constant velocity model (in dark red), and for multi-layer tomography (in light blue) overlaying the combined high-resolution, high-definition+multi-layer tomography (in dark blue) which achieves the best QI score. The high-resolution velocity models (in blue) converge faster during seismic inversion to a higher QI score as the synthetic traces generated during the forward-modelling stage of the inversion match the actual traces more closely. These high-resolution models are therefore a better approximation of the subsurface as they require fewer changes to match the observed seismic traces.
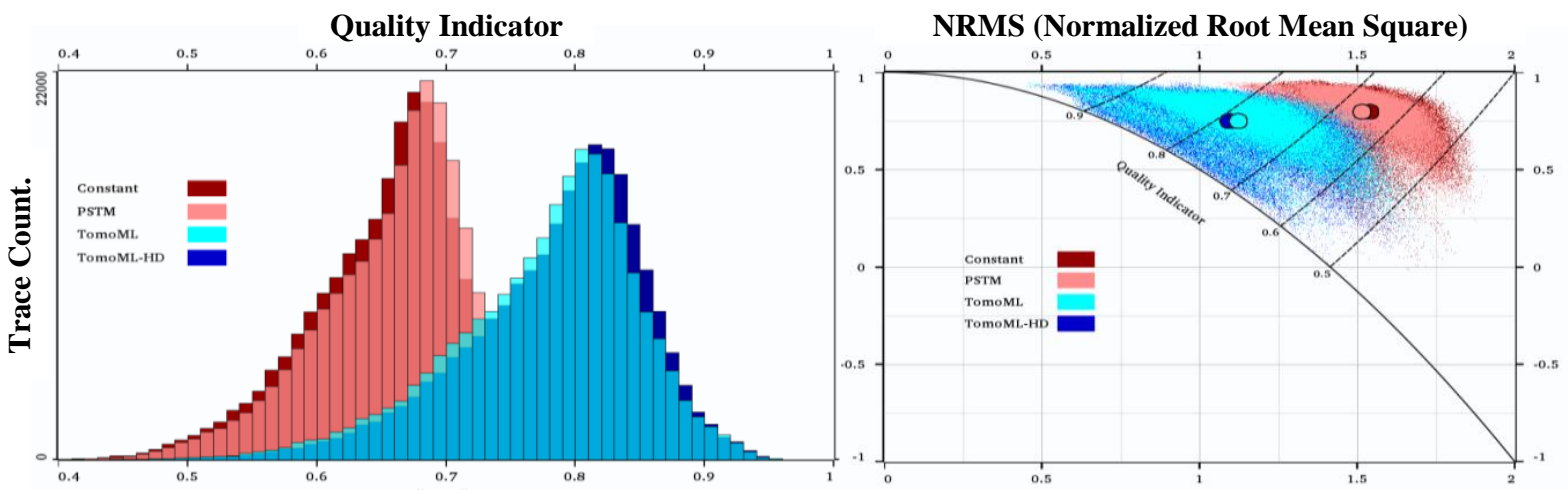

Figure 3: Comparison of synthetic to seismic trace matching statistics from different initial models during seismic inversion: Quality Indicator (QI) measurements per trace in histogram (left) and in a Correlation-NRMS cross-plot (right).

\section{Conclusions}

New ray based tomographic methods provide velocity models with a high resolution and with an accurate positioning of the major velocity contrasts. They can be used as attributes for reservoir characterization increasing the accuracy of the initial model, and so reducing the non-uniqueness of the inverse problem and reducing the uncertainties for quantitative prediction of rock properties.

\section{Acknowledgements}

The authors thank CGG for the authorization to present this work, and GDF Suez E\&P Nederland B.V. and their partners for their permission to show the case study. The authors are also grateful to their colleagues in the Imaging and Reservoir teams for the collective effort in preparing these results.

\section{References}

- Claerbout J.K., 1985. Imaging the Earth's Interior, Copyright @ 1984 by The Board of Trustees of the Leland Stanford Junior University Stanford, California 94305, U.S.A. http://sep.stanford.edu/sep/prof/iei2/ .

- Coléou T., Coulon J-P., Carotti D., Dépré P., Robinson G., Hudgens E., 2013. AVO QC during processing, Extended abstracts, $75^{\text {th }}$ EAGE Conference \& Exhibition, London.

- Guillaume, P., G. Lambaré, S. Sioni, X. Zhang, A. Prescott, D. Carotti, P. Dépré, S. Frehers and H. Vosberg, 2012. Building detailed structurally conformable velocity models with high definition tomography, $74^{\text {th }}$ EAGE Conference \& Exhibition, W002.

- Guillaume, P., X. Zhang, A. Prescott, G. Lambaré, M. Reinier, J.-P. Montel, A. Cavalié, 2013. Multi-layer non-linear slope tomography, $75^{\text {th }}$ EAGE Conference \& Exhibition, Th-04-01. 